\title{
Correspondence
}

\section{Physostigmine in 1999: tolerability, indications and availability}

To the Editor:

The journal recently reported a randomized doubleblinded placebo-controlled study showing antagonism of propofol anesthesia by physostigmine, ${ }^{1}$ in which the authors presented only a limited discussion of the clinical relevance. I believe that this reflects the current reluctance of anesthesiologists to use physostigmine, as well as an under-recognition of its beneficial properties. Not surprisingly, North America no longer has a readily available supply of this drug.

In the reported investigation, the absence of undesired cholinergic effects should be emphasized: the randomized administration of $2 \mathrm{mg}\left(0.033 \mathrm{mg} \cdot \mathrm{kg}^{-1}\right)$ physostigmine iv five minutes before the induction of propofol anesthesia in $\mathbf{2 0}$ unpremedicated adult women was not associated with bradycardia, bronchospasm, nausea or vomiting. A tolerability profile similar to placebo has also been reported following anesthesia in 170 patients in various randomized clinical trials investigating the antisedative, ${ }^{2,3}$ antishivering ${ }^{4}$ and analgesic ${ }^{5}$ properties of 2-3 $\mathrm{mg}\left(0.03-0.04 \mathrm{mg} \cdot \mathrm{kg}^{-1}\right)$ physostigmine.

Physostigmine is still the drug of choice in treating postanesthetic abnormal awakening, whose incidence without scopolamine premedication is reportedly as high as $1.9 \%{ }^{6}$ After excluding nonpharmacological disturbances, a positive response to physostigmine confirms a diagnosis of "central anticholinergic syndrome." Accordingly, physostigmine may relieve prolonged postanesthetic somnolence as well as agitated behaviours such as delirium, ${ }^{7}$ seizure-like movements ${ }^{8}$ and opisthotonos. ${ }^{9}$

Should physostigmine be re-stocked in every postanesthetic recovery area? To answer this question, compare the occasional cost of intensive nursing care, specialist physician consultation and patient morbidity when prolonged abnormal awakening remains untreated to the annual cost of maintaining a supply of physostigmine. In my view, a cost-benefit analysis would indicate that your hospital pharmacy should be asked to provide physostigmine for emergency use during anesthetic recovery.

Craig R. Ries MD FRCPC PhD

Vancouver, B.C.

\section{References}

1 Fassoulaki $A$, Sarantopoulos $C$, Derveniotis $C$. Physostigmine increases the dose of propofol required to induce anaesthesia. Can J Anaesth 1997; 44: 1148-51.

2 Kesecioglu J, Rupreht, Telci L, Dzoljic M, Erdmann W. Effect of aminophylline or physostigmine on recovery from nitrous oxide-enflurane anaesthesia. Acta Anaesthesiol Scand 1991; 35: 616-20.

3 Hill GE, Stanley TH, Sentker CR. Physostigmine reversal of postoperative somnolence. Can Anaesth Soc J 1977; 24: 707-11.

4 Horn E-P, Standl T, Sessler DI, von Knobelsdorff G, Büchs $C$, Schulte am Esch J. Physostigmine prevents postanesthetic shivering as does meperidine or clonidine. Anesthesiology 1998; 88: 108-13.

5 Petersson J, Gordh, TE, Hartvig P, Wiklund L. A double-blind trial of the analgesic properties of physostigmine in postoperative patients. Acta Anaesthesiol Scand 1986; 30: 283-8.

6 Link J, Papadopoulos G, Dopjans D, GuggenmoosHolzmann $I$, Eyrich $K$. Distinct central anticholinergic syndrome following general anaesthesia. Eur J Anaesthesiol 1997; 14: 15-23.

7 Weinger $M B$, Swerdlow NR, Millar WL. Acute postoperative delirium and extrapyramidal signs in a previously healthy parturient. Anesth Analg 1988; 67: 291-5.

8 Kaiser-Stadler $M$, Altmayer P. Central anticholinergic syndrome after propofol anesthesia. (German) Anasthesiol Intensivmed Notfallmed Schmerzther 1995; 30: 116-7.

9 Debring DJ, Gupta B, Peruzzi WT. Postoperative opisthotonus and torticollis after fentanyl, enflurane, and nitrous oxide. Can J Anaesth 1991; 38: 919-25.

\section{Gastroparesie d'origine centrale: utilite de l'erythromycine}

L'érythromycine est douée d'une action prokinétique au niveau gastrique, connue et utilisée depuis plusieurs années pour la prise en charge de certaines gastroparésies accompagnant la maladie diabétique. ${ }^{1}$ D'autres applications ont été décrites de façon ponctuelle dans la littérature, comme dans les suites de vagotomie. $^{2}$

Nous relatons notre expérience à propos d'un 